\title{
Two rare cases of synchronous and metachronous colonic metastases in patients with advanced gastric cancer
}

\author{
Wei-Chih Su ${ }^{1,2}$, Hsiang-Lin Tsai ${ }^{1,3}$, Chun-Chieh Wu ${ }^{4}$, Shan-Yin Tsai ${ }^{4}$, Yung-Sung Yeh ${ }^{1,2,5}$, Cheng-Jen Ma,6 \\ and Jaw-Yuan Wang ${ }^{1,2,3,7,8,9,10^{*}}$
}

\begin{abstract}
Background: Patients with advanced gastric cancer (GC) may ultimately die because GC mostly leads to synchronous or metachronous metastasis. However, colonic metastasis of GC is extremely rare. According to a PubMed search of papers published from May 1968 to March 2017, only 21 patients with GC (10 patients from 10 case reports and 11 patients from a retrospective study) have been found to have colonic metastasis. In this report, we present two cases of synchronous and metachronous colonic metastases of advanced GC.

Case presentation: Two patients with advanced GC received a diagnosis of colonic metastasis based on colonoscopic findings and computed tomography images, and the diagnosis was confirmed through pathological immunohistochemical analysis. Herein, we describe the management and outcomes of these metastases.
\end{abstract}

Conclusions: Submucosal swelling and segmental bowel wall thickening observed through colonoscopy in patients with advanced GC might indicate colonic metastasis.

Keywords: Gastric cancer, Synchronous colonic metastasis, Metachronous colonic metastasis

\section{Background}

Gastric carcinoma is the forth leading cause of cancerrelated deaths worldwide [1]. In the advanced stages of this disease, patients may develop either synchronous or metachronous metastasis. The most common sites of gastric cancer (GC) metastasis are the liver, peritoneum, lungs, and bones [2]. According to our review of the literature published between May 1968 and March 2017, 21 patients with GC (10 patients from 10 case reports and 11 patients from a retrospective study) received a diagnosis of colonic metastasis [3-13]. In this report, we present two rare cases of synchronous and metachronous colonic metastases of advanced GC and report their diagnoses, management, and clinical outcomes.

\footnotetext{
* Correspondence: cy614112@ms14.hinet.net; jayuwa@cc.kmu.edu.tw 'Division of Colorectal Surgery, Department of Surgery, Kaohsiung Medical University Hospital, No. 100, Tzyou 1st Road, Kaohsiung 807, Taiwan ${ }^{2}$ Graduate Institute of Clinical Medicine, College of Medicine, Kaohsiung Medical University, Kaohsiung, Taiwan

Full list of author information is available at the end of the article
}

\section{Case presentation}

Case 1: synchronous colonic metastasis of advanced GC A 77-year-old man visited our emergency department with acute abdominal pain for 2 days. After initial evaluation, abdominal computed tomography (CT) was performed, which revealed diffuse wall edema of the rectosigmoid colon (Fig. 1a). Rectosigmoid colon cancer with partial obstruction was suspected, and transverse colostomy was subsequently performed. Colonoscopy revealed mucosal swelling on the anal side a few days after the stool diversion procedure (Fig. 1b). Pathological examination of the colonoscopic biopsy revealed only chronic inflammation. The second abdominal CT examination, performed after 1.5 months, showed circumferential thickening of the pylorus with marked stomach distension (Fig. 1c), and advanced GC was tentatively diagnosed. Esophagogastroduodenoscopy revealed a hyperemic mucosal lesion over the antrum, and the pathology report revealed poorly differentiated GC. Colonoscopy was performed 


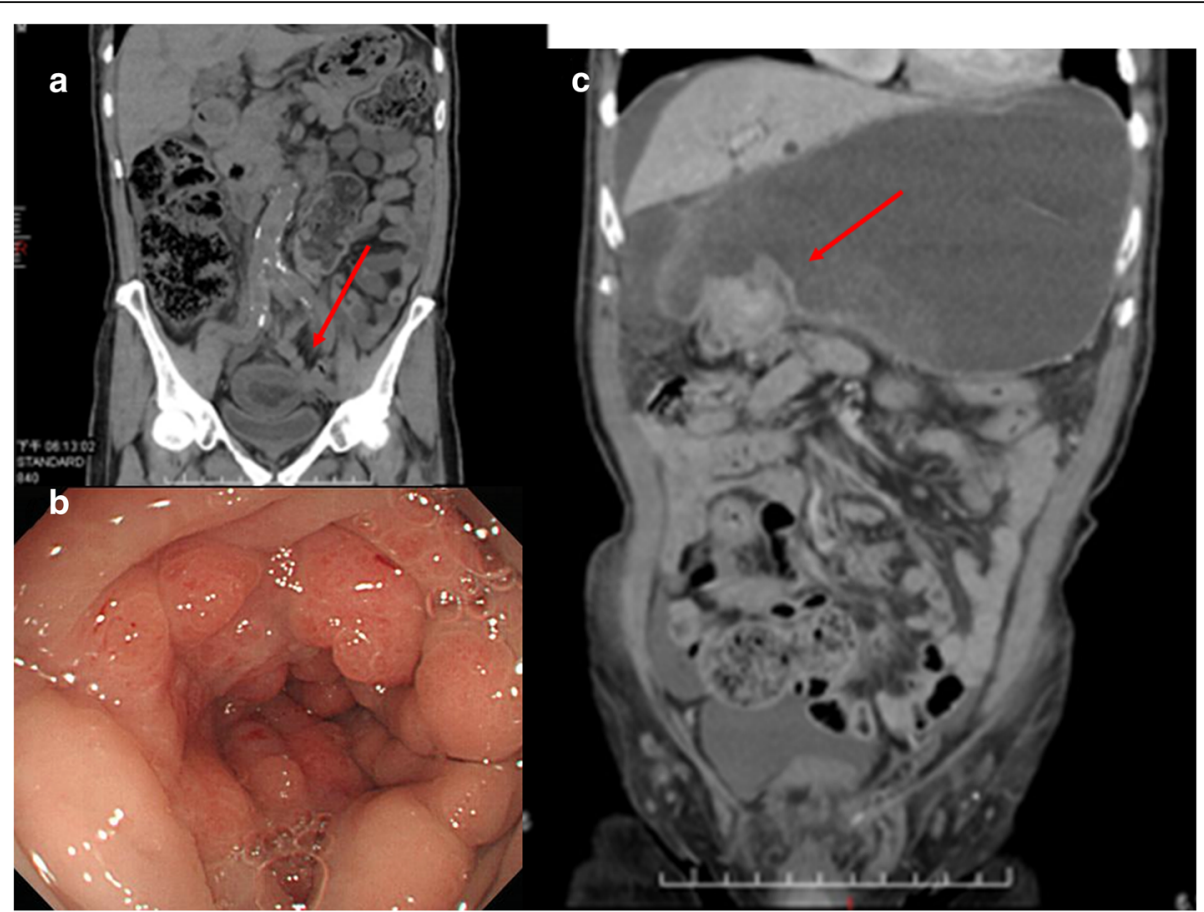

Fig. 1 Synchronous colonic metastasis of advanced GC. a Abdominal CT showed suspected rectosigmoid colon cancer with partial obstruction; b colonoscopy revealed rectosigmoid colon wall thickening and partially obstructed mucosal swelling; c abdominal CT displayed circumferential thickening of the pylorus and marked stomach distension, indicating the possibility of GC

again, and the second pathology report confirmed poorly differentiated GC with colonic submucosal involvement (Fig. 2a). The immunohistochemical analysis results were as follows: CDX-2 (-) (Fig. 2b), CK20 (-) (Fig. 2c), and CK7 (+) (Fig. 2d). The final pathology report stated that the patient had advanced GC with synchronous colonic metastasis. Although the patient received neoadjuvant chemotherapy of 7 cycles of oxaliplatin, folinic acid, and 5-fluorouracil (FOLFOX4) regimen for disease control, he survived for only 6 months.

Case 2: metachronous colonic metastasis of advanced GC A 78-year-old man had advanced GC with poorly differentiated histology (pT3N3aM0). Eighteen months previously, radical subtotal gastrectomy and Billroth-II gastrojejunostomy had been performed. In addition, he had received neoadjuvant therapy of 12 cycles of the FOLFOX4 regimen. Because the patient had developed progressive abdominal distension, abdominal CT was performed, which showed a circumscribed mass lesion with severe distention at the proximal colonic loop. CT scans also revealed a small bowel loop, which was suspected to be a colon tumor (Fig. 3a). Colonoscopy revealed mucosal swelling characterized by a completely obstructive lesion (Fig. 3b). Transverse colectomy and end-to-end colocolostomy were performed for confirmation.
The pathology report confirmed that the patient had metachronous colonic metastasis of advanced GC (Fig. 4a). Immunohistochemical analysis obtained the following marker results: CDX-2 (weak and faintly positive) (Fig. 4b), CK20 (-) (Fig. 4c), and CK7 (+) (Fig. 4d). However, the clinical outcome was negative owing to rapid disease progression. The patient survived for only 6 months after receiving the diagnosis.

\section{Discussion and conclusions}

Colonoscopy revealed that the two patients exhibited different manifestations of colonic masses. We observed only wall thickening and swelling in both cases, with no signs of contact bleeding or centrally ulcerated lesions of the colon. These findings are different from the classic appearance of primary colon cancer upon colonoscopy. Furthermore, intestinal metastasis of GC has rarely been reported [14].

Jang et al. performed a retrospective radiological analysis of $23 \mathrm{GC}$ patients with intestinal metastasis, of whom only 11 were pathologically diagnosed as having colonic metastasis. Most of the patients had poorly differentiated adenocarcinoma or the signet-ring cell type with a propensity to develop into rare intestinal metastasis. Notably, the two patients in the present case report had poorly differentiated adenocarcinoma. 

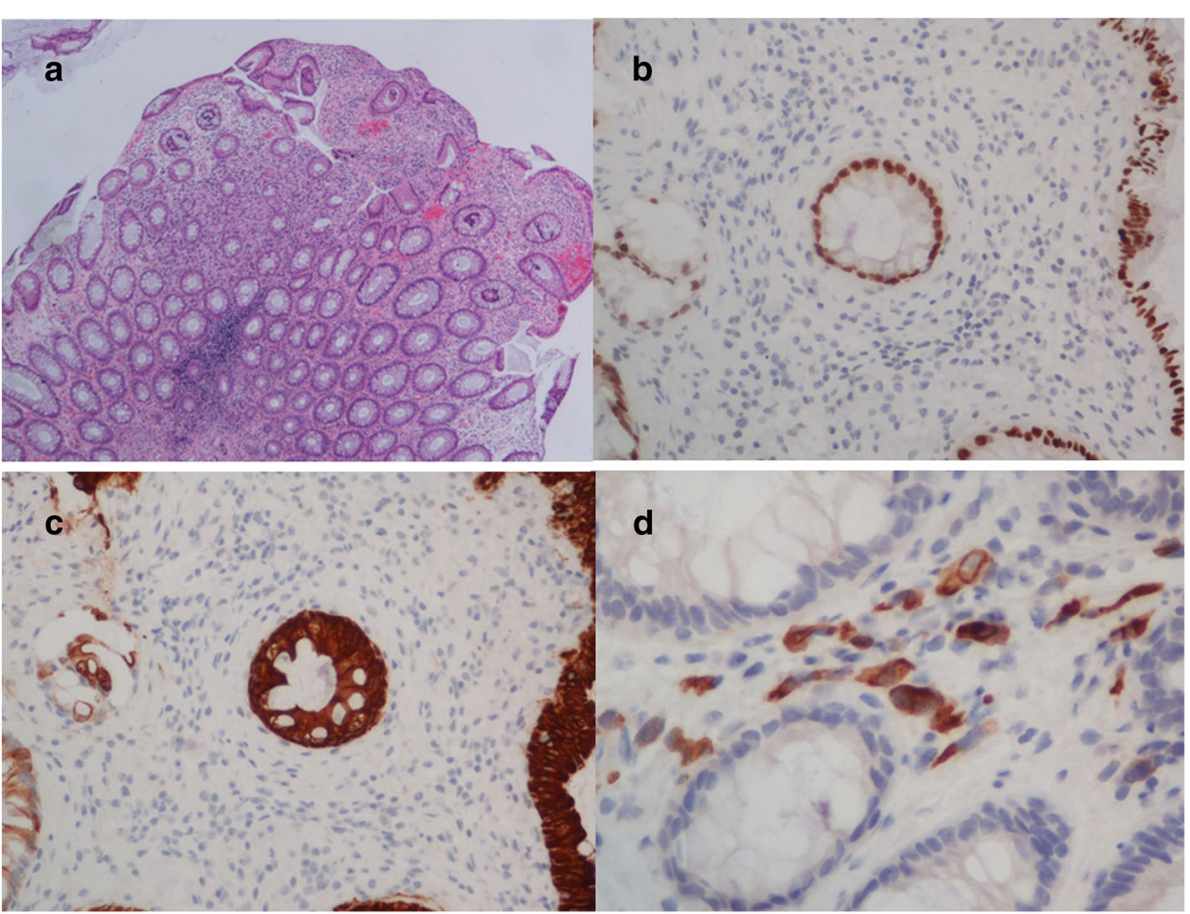

Fig. 2 a Poorly differentiated carcinoma involving colonic submucosa hematoxylin and eosin staining (HE) $(\times 40)$; b CDX-2 staining with negative results ( $\times 100)$; c CK20 staining with negative results ( $\times 100)$; and $\mathbf{d}$ CK7 staining with positive results $(\times 400)$

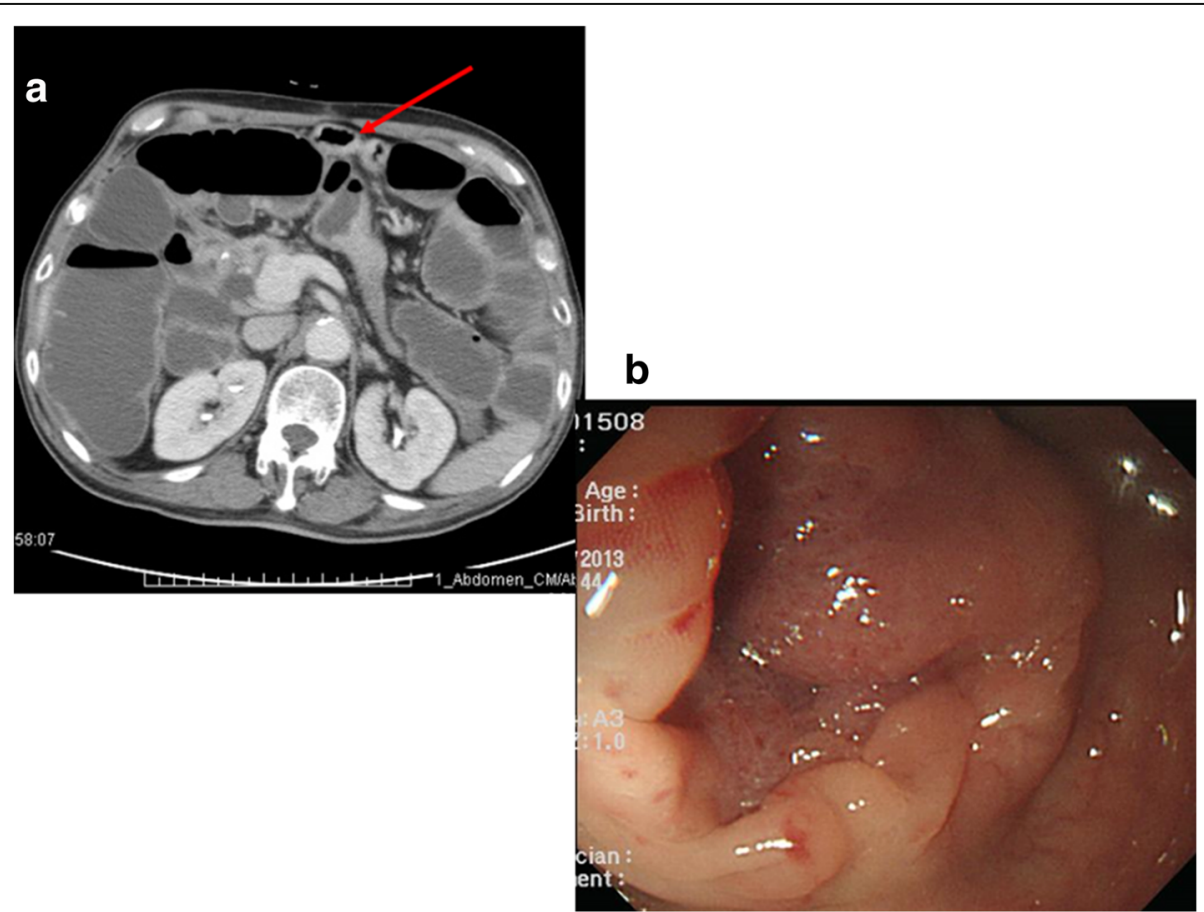

Fig. 3 Metachronous colonic metastasis of advanced GC. a Abdominal CT showed a circumferential mass lesion with severe distention of the proximal colonic loop and small bowel loop, suggesting colonic metastasis. b Colonoscopy revealed mucosal swelling with total obstruction 


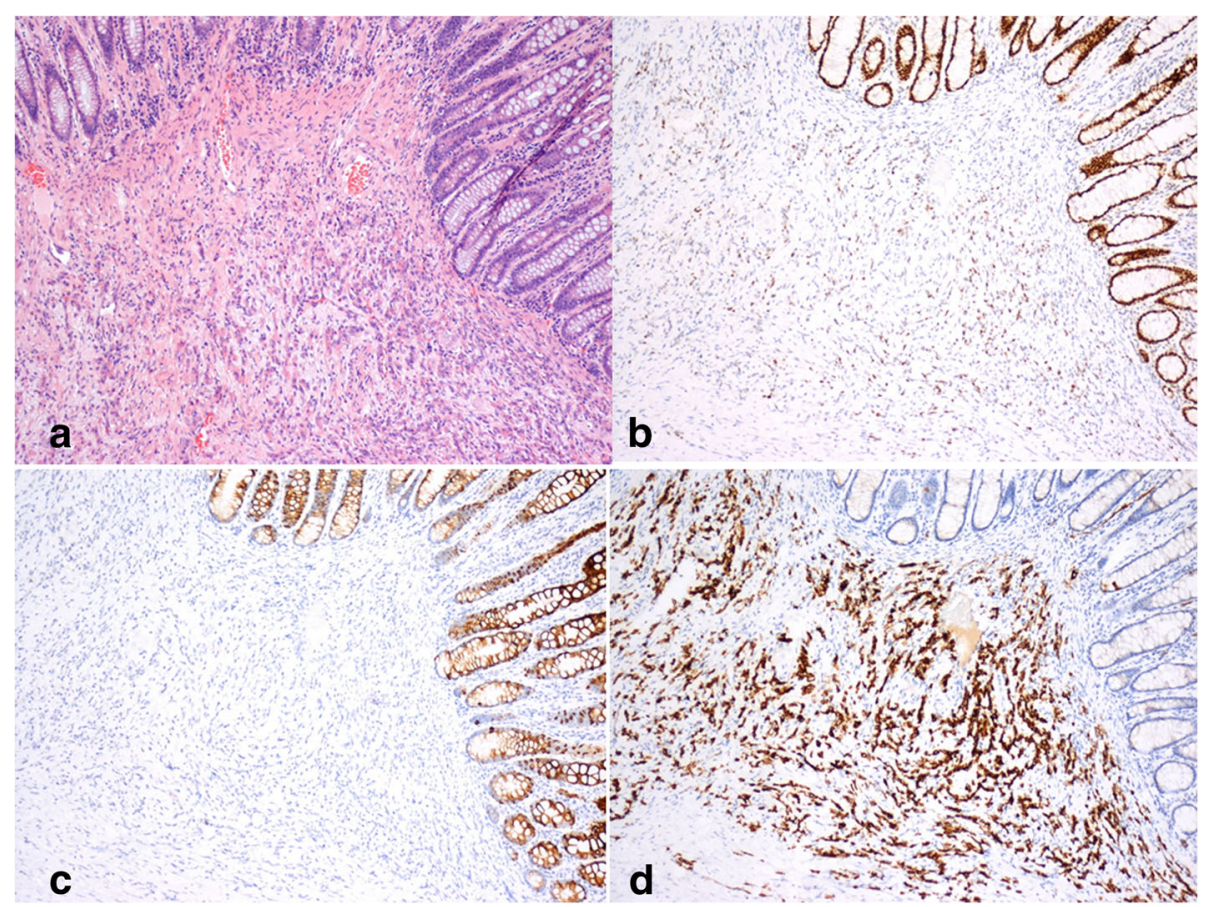

Fig. 4 a Poorly differentiated carcinoma involving colonic submucosa HE $(\times 40)$; b CDX-2 staining with weak and faintly positive results $(\times 100)$; c CK20 staining with negative results $(\times 100)$; and $\mathbf{d}$ CK7 staining with positive results $(\times 400)$

Furthermore, intestinal metastasis should be considered by physicians for patients with GC who exhibit wall thickening over segmental bowel and those who exhibit target enhancement and progressive thickening of the enhancing inner layer on CT images [13, 15].

In our cases, segmental bowel wall swelling and progressive thickening of the enhancing inner layer were detected through colonoscopy and CT, respectively. The final diagnosis of colonic metastasis was based on histological features and immunohistochemical analysis. The analyses confirmed the expression of negative CDX-2 in one case, weak and faintly positive CDX-2 in the other, and negative CK20 in both [16, 17]. Moreover, positive CK7 staining was performed to exclude the possibility of prostate cancer.

Cases of GC with colonic metastasis are extremely rare. The survival period for most established cases ranges from 1 to 10 months [4,9-11]; both patients in our report survived for approximately 6 months after their diagnosis. Furthermore, the prognosis of advanced $\mathrm{GC}$ in the two cases was relatively poor even after aggressive treatment. Therefore, colonic metastasis should be considered by physicians if colonoscopy reveals submucosal swelling and segmental bowel wall thickening in patients with advanced GC, particularly in those with poorly differentiated adenocarcinoma or the signet-ring cell type.

\section{Abbreviations}

CT: Computed tomography; FOLFOX4: Oxaliplatin, folinic acid, and 5-fluorouracil; GC: Gastric cancer

\section{Acknowledgements \\ This manuscript was edited by Wallace Academic Editing.}

\section{Funding}

This work was supported by grants from the Excellence for Cancer Research Center through funding by the Ministry of Science and Technology (MOST1052325-B-037-001, MOST106-2314-B-037-019-) and the Ministry of Health and Welfare (MOHW106-TDU-B-212-144007); Health and Welfare Surcharge of Tobacco Products, Taiwan, Republic of China; and Kaohsiung Medical University Hospital (KMUH104-4M25, KMUH104-4M51, KMUH105-5M21, KMUH106-6R32, KMUH1066M28, KMUH106-6M29, KMUH106-6M30, KMUH106-6M31, KMUHS10522, KMUHS10505, KMUHS10518, and KMUHGCRC2016002, KMUHS10601, KMUHS10608, KMUHA10664). In addition, this study was supported by Kaohsiung Medical University "Aim for the Top 500 Universities Grant" (KMUTP105C01, KMU-TP105C11) Kaohsiung, Taiwan; "Aim for the Top University Grant," under grant nos. KMU-S105011, KMU-TP105A14, KMU-DK106005, and SH000113 (Give2Asia); and the Grant of Biosignature in Colorectal Cancers (grant no.T107-001), Academia Sinica, Taiwan.

\section{Availability of data and materials}

All findings of this case report are based on diagnostic examinations performed during patient hospitalization. The publication of these data was authorized by Kaohsiung Medical University Hospital. Data sharing is not applicable to this article as no datasets were generated or analyzed in the present report.

\section{Authors' contributions}

SWC wrote the original manuscript. WCC and TSY interpreted the pathological findings. The final manuscript was revised by THL, YYS, MCJ, and WJY. The final manuscript has been read and approved by all authors. 


\section{Ethics approval and consent to participate}

This study was approved by the Institutional Review Board of Kaohsiung Medical University Hospital (KMUH-IRB 1217-1417)

\section{Consent for publication}

Not applicable as this is a retrospective review of two expired patients.

\section{Competing interests}

The authors declare that they have no competing interests.

\section{Publisher's Note}

Springer Nature remains neutral with regard to jurisdictional claims in published maps and institutional affiliations.

\section{Author details}

'Division of Colorectal Surgery, Department of Surgery, Kaohsiung Medical University Hospital, No. 100, Tzyou 1st Road, Kaohsiung 807, Taiwan. ${ }^{2}$ Graduate Institute of Clinical Medicine, College of Medicine, Kaohsiung Medical University, Kaohsiung, Taiwan. ${ }^{3}$ Department of Surgery, Faculty of Medicine, College of Medicine, Kaohsiung Medical University, Kaohsiung, Taiwan. ${ }^{4}$ Department of Pathology, Kaohsiung Medical University Hospital, Kaohsiung, Taiwan. ${ }^{5}$ Division of Trauma and Surgical Critical Care, Department of Surgery, Kaohsiung Medical University Hospital, Kaohsiung Medical University, Kaohsiung, Taiwan. ${ }^{6}$ Division of General and Digestive Surgery, Department of Surgery, Kaohsiung Medical University Hospital, Kaohsiung Medical University, Kaohsiung, Taiwan. ${ }^{7}$ Graduate Institute of Medicine, College of Medicine, Kaohsiung Medical University, Kaohsiung, Taiwan. ${ }^{8}$ Center for Biomarkers and Biotech Drugs, Kaohsiung Medical University, Kaohsiung, Taiwan. ${ }^{9}$ Research Center for Environmental Medicine, Kaohsiung Medical University, Kaohsiung, Taiwan. ${ }^{10}$ Research Center for Natural Products \& Drug Development, Kaohsiung Medical University, Kaohsiung, Taiwan.

Received: 30 May 2017 Accepted: 23 January 2018

\section{Published online: 31 January 2018}

\section{References}

1. World Health Organization. Cancer: Fact Sheet No 297. WHO. Available at http://www.who.int/mediacentre/factsheets/fs297/en/. Accessed 24 Apr 2017.

2. Riihimäki $M$, Hemminki $A$, Sundquist $K$, Sundquist J, Hemminki K. Metastatic spread in patients with gastric cancer. Oncotarget. 2016;7(32):52307-16.

3. Lee IH, Lee JE, Byeon SW, Lee HJ, Huo SM, Yoon SB, et al. A case of advanced gastric cancer presenting as multiple colonic lymphoid hyperplasia. Korean J Gastroenterol. 2015;66(4):221-6. [Article in Korean]

4. Gao B, Xue X, Tai W, et al. Polypoid colonic metastases from gastric stump carcinoma: a case report. Oncol Lett. 2014;8(3):1119-22.

5. Metayer $\mathrm{P}$, Antoneitti M, Oumrani $\mathrm{M}$, et al. Metastases of a gastric adenocarcinoma presenting as colonic polyposis. Report of a case. Dis Colon Rectum. 1991;34:622-3.

6. Ogiwara $\mathrm{H}$, Konno H, Kitayama $\mathrm{Y}$, et al. Metastases from gastric adenocarcinoma presenting as multiple colonic polyps: report of a case. Surg Today. 1994;24:473-5.

7. Tiszlavicz L. Stomach cancer metastasizing into a solitary adenomatous colonic polyp. Orv Hetil. 1990;131:1259-61. [Article in Hungarian]

8. Dohden $\mathrm{K}$, et al. Metastases from gastric carcinoma to esophagus, duodenum and large intestine in the form of polyposis, report of a case. Stomach Intestine (Tokyo). 2002;37:1238-42. [Article in Japanese]

9. Lee HC, Yang MT, Lin KY, Tu HY, Zhang TA, Chen PH. Metastases from gastric carcinoma to colon in the form of multiple flat elevated lesions: a case report. Kaohsiung J Med Sci. 2004;20:552-7.

10. Nakamura H, Fu K, Fukui H, Hurlstone DP, Kaji Y, Ishikawa T, Fujimori T. A solitary colonic metastasis from gastric cancer detected at an early stage. Gastrointest Endosc. 2008;67(6):1000-4.

11. Pace U, Contino G, Chiappa A, Bertani E, Bianchi PP, Facio N, et al. Metachronous colon metastases from gastric adenocarcinoma: a case report. Case Reports in Oncology. 2009;2(2):92-6.

12. Fujimoto D, Hirono Y, Goi Y, Yamaguchi A. Sigmoid colonic metastasis by lymphatic spread occurring with unilateral Krukenberg tumor considered to be caused by stage IA early gastric cancer: a case report. Oncol Lett. 2016;11(1):668-72.
13. Jang HJ, Lim HK, Kim HS, et al. Intestinal metastases from gastric adenocarcinoma: helical CT findings. J Comput Assist Tomogr. 2001;25:61-7.

14. Duarte I, Llanos O. Pattern of metastases in intestinal and diffuse types of carcinoma of the stomach. Hum Pathol. 1981;12:237-42.

15. Fernandes T, Oliveira MI, Castro R, Araújo B, Viamonte B, Cunha R. Bowel wall thickening at $C T$ : simplifying the diagnosis. Insights Imaging. 2014;5(2):195-208.

16. Werling RW, Yaziji $H$, Bacchi CE, et al. CDX2, a highly sensitive and specific marker of adenocarcinomas of intestinal origin. Am J Surg Pathol. 2003;27:303-10.

17. Bayrak R, Haltas H, Yenidunya S. The value of CDX2 and cytokeratins 7 and 20 expression in differentiating colorectal adenocarcinomas from extraintestinal gastrointestinal adenocarcinomas: cytokeratin 7-/20+ phenotype is more specific than CDX2 antibody. Diagn Pathol. 2012;7:9.

\section{Submit your next manuscript to BioMed Central and we will help you at every step:}

- We accept pre-submission inquiries

- Our selector tool helps you to find the most relevant journal

- We provide round the clock customer support

- Convenient online submission

- Thorough peer review

- Inclusion in PubMed and all major indexing services

- Maximum visibility for your research

Submit your manuscript at www.biomedcentral.com/submit
) Biomed Central 